\title{
Gap Opening in Ultrathin Si Layers: Role of Confined and Interface States
}

\author{
Stefano Ossicini, A. Fasolino, and F. Bernardini \\ Dipartimento di Fisica, Università di Modena, Via Campi 213/A, 41100 Modena, Italy
}

(Received 22 July 1993)

\begin{abstract}
We present first principle calculations of ultrathin silicon (111) layers embedded in $\mathrm{CaF}_{2}$, a lattice matched insulator. Our all electron calculation allows a check of the quantum confinement hypothesis for the Si band gap opening as a function of thickness. We find that the gap opening is mostly due to the valence band while the lowest conduction band states shift very modestly due to their pronounced interface character. The latter states are very sensitive to the sample design. We suggest that a quasidirect band gap can be achieved by stacking Si layers of different thickness.

PACS numbers: 68.35.-p, 68.65.+g, 71.25.Tn, 73.20.Dx
\end{abstract}

The observation of bright, visible light emission from porous $\mathrm{Si}$ [1] has stimulated a wealth of experimental [2] and theoretical work [3-12] to understand both the energy shift and the increased efficiency of luminescence in porous $\mathrm{Si}$ and in laterally confined $\mathrm{Si}$ structures, with respect to bulk Si. Bulk Si has a $1.1 \mathrm{eV}$ indirect gap resulting in luminescence in the near infrared with very low efficiency.

All theoretical approaches, ranging from effective mass [3] to tight binding [4-7] to $a b$ initio local density approximation (LDA) treatments [8-12], give a qualitative account of the shift of the band gap from the near infrared to, and beyond, the visible range as due to confinement and an enhancement of the dipole matrix element for radiative transitions resulting from the better overlap of confined wave functions. However, a complete understanding of the problem is far from being reached. In particular, the recent observation of phonon satellites in the photoluminescence of porous silicon [13] questions the hypothesis of a direct gap as the origin of the increased luminescence efficiency.

The main problem for a quantitative description of porous $\mathrm{Si}$ is the still not characterized microscopic structure and shape of the crystallites. This is why we have chosen to address the problem of $\mathrm{Si}$ crystallites embedded in $\mathrm{CaF}_{2}$, as prototype of a Si-based system with known microscopic structure [14-17] for which evidence of visible luminescence has been claimed [18]. We show that the energy spectrum of $\mathrm{Si}$ layers is affected both by confinement, yielding a blueshift of the gap, and by hybridization effects with the saturating agent, be it the $\mathrm{Ca}$ atoms in our case and $\mathrm{H}$ or $\mathrm{O}$ in porous silicon, leading to a high joint density of states all over the Brillouin zone (BZ). By use of a new procedure, we are able to assign the blueshift of the $\mathrm{Si}$ gap in the $\mathrm{CaF}_{2} / \mathrm{Si} / \mathrm{CaF}_{2}$ system mostly to the valence band. Our results are compatible with the observation of visible luminescence in this system, since the band gap energy is found to increase for decreasing Si thickness. Moreover, we suggest that the band gap can be made quasidirect by stacking, in the $\mathrm{CaF}_{2}$ matrix, Si layers of different thickness.
We present first principle calculations, performed by the linear muffin-tin orbital method in the atomic-sphere approximation (LMTO-ASA) within the LDA, of the band structure of thin [1-7 double layers (DL)] $\mathrm{Si}(111)$ slabs embedded in $\mathrm{CaF}_{2}$. The LMTO-ASA method correctly describes the $\mathrm{Si} / \mathrm{CaF}_{2}$ interface properties $[19,20]$. Because of the LDA we underestimate the energy gap: we obtain 0.56 and $6.96 \mathrm{eV}$ instead of 1.1 and $12.1 \mathrm{eV}$ for bulk $\mathrm{Si}$ and $\mathrm{CaF}_{2}$, respectively.

Crystalline $\mathrm{CaF}_{2}$ and $\mathrm{Si}$ have similar fcc structures, with a room temperature lattice mismatch of $0.6 \%$. High quality epitaxial fluoride layers can be grown on $\mathrm{Si}(111)[14,15]$; in the reverse process, $\mathrm{Si}$ on $\mathrm{CaF}_{2}$, the natural growth mode would be island formation. This difficulty can be overcome by electron irradiation [15] or by low temperature $\mathrm{Si}$ deposition followed by thermal treatment in order to recrystallize it [21]. The interface structure is shown in Fig. 1. The first monolayer of $\mathrm{CaF}_{2}$ loses half of its fluorine atoms leading to a $\mathrm{Ca}-\mathrm{Si}$ bond at the interface. The interface $\mathrm{Ca}$ atoms occupy the $T_{4}$ sites, the triangular filled sites on top of the second layer $\mathrm{Si}$ atoms, while the $\mathrm{F}$ atoms are located on the $H_{3}$ sites, the triangular hollow sites on top of the fourth layer $\mathrm{Si}$ atoms.

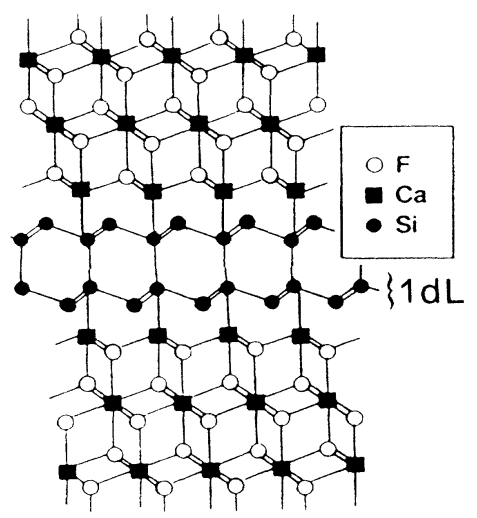

FIG. 1. Structural model for the $\mathrm{CaF}_{2}-\mathrm{Si}-\mathrm{CaF}_{2}$ system; 1 DL is indicated. 
We use supercells formed by thin $\mathrm{Si}$ layers of variable thickness and by $\mathrm{CaF}_{2}$ layers large enough to make the central $\mathrm{CaF}_{2}$ planes exhibit bulklike properties. We use throughout the lattice constant of $\mathrm{CaF}_{2}(a=5.462 \AA)$ except for the interlayer $\mathrm{Si}-\mathrm{Ca}$ distance, which is taken to be $2.36 \AA$, as determined by $\mathrm{x}$-ray standing waves experiments [16], $0.1 \AA$ longer than the one estimated by medium energy ion scattering [17]. The theoretical results are not significantly affected by such a difference [20].

The LMTO-ASA applied to the $\mathrm{Si} / \mathrm{CaF}_{2}$ system allows one to compare on a single energy scale the band structure calculated for different $\mathrm{Si}$ thicknesses, since the $2 s$ core level of the central fluorine atom can be used as reference energy. In the following, we will justify the assumption that the fluorine $2 s$ core level remain at the same energy for all $\mathrm{Si}$ layer thicknesses. As shown in Fig. 2, this procedure allows one to follow separately the shift of the conduction and valence bands in going from 7 to $1 \mathrm{DL}$. The band structure, in the energy region around the gap, is displayed along the $\Gamma-M$ direction of the hexagonal two-dimensional BZ of the (111) surface, i.e., the direction where the bulk $\Gamma-X$ direction, where the minimum of the Si conduction band occurs, is now projected.

It can be seen that the gap opening is dominated by the Si valence band, which shifts to lower energies for thinner layers. Therefore, quantum confinement of the valence band accounts for the greatest part of the blueshift of the gap. In particular, the energy shift with layer thickness compares very well with that calculated within the effective mass theory, by taking the heavy hole mass as $0.281 m_{0}$ for bulk $\mathrm{Si}, 0.3 m_{0}$ for $\mathrm{CaF}_{2}$, and a valence band offset of $6 \mathrm{eV}$, values estimated from LMTO calculations.

The lowest conduction band level, instead, is dominated by hybridization effects. It is mostly localized at the $\mathrm{Si}-\mathrm{Ca}$ interface and therefore it is much flatter than the bulk conduction band and rather insensitive to the Si thickness. The presence of this interface state has the interesting consequence of keeping the growth of the gap limited to much lower energies than expected from complete quantum confinement as in the case of $\mathrm{H}$-saturated Si structures [8]. This state represents the antibonding state due to the Si-Ca bond: its bonding partner is buried in the $\mathrm{Si}$ valence band for the thicker layers and emerges, for thinner layers, from the $\mathrm{Si}$ valence band to become the highest occupied level. In particular, at $\Gamma$, the bonding interface state has an upward curvature, since it results from hybridization of the $s-d$ states of the interface $\mathrm{Ca}$ atoms (which constitute the conduction band of $\mathrm{CaF}_{2}$ ) with the valence $p$ states of Si. It should be noted that the $\mathrm{Si}-\mathrm{Ca}$ bond at the interface is somewhat intermediate between the covalent $\mathrm{Si}-\mathrm{Si}$ bond and the ionic $\mathrm{Ca}$ $\mathrm{F}$ bond. Therefore, the bonding-antibonding states are not removed from the gap as in the case of $\mathrm{H}$-saturated $\mathrm{Si}$ structures. The H-Si bond, being mostly covalent,

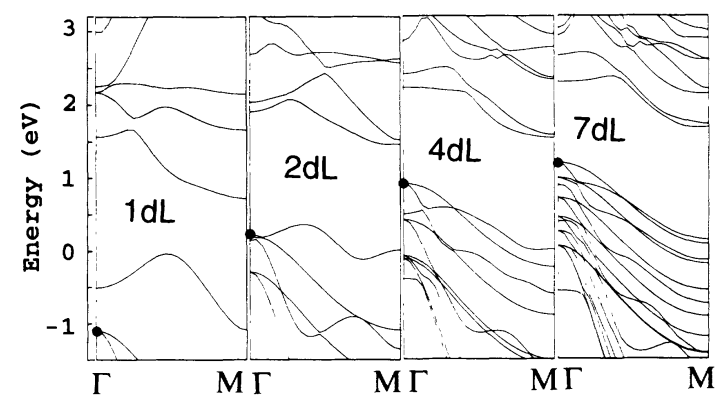

FIG. 2. Band structures along the $\Gamma-M$ symmetry direction of the two-dimensional hexagonal (111) $\mathrm{BZ}$ on a single energy scale for $1,2,4$, and $7 \mathrm{DL} \mathrm{Si}$ in $\mathrm{CaF}_{2}$ (see text). The band gap opening is dominated by the confinement induced shift of the valence band of $\mathrm{Si}$ evidenced by dots.

gives rise instead to a larger bonding-antibonding energy separation and pushes the interface states inside the $\mathrm{Si}$ valence band and above the conduction band minimum $[8-12,22,23]$.

In order to prove that the new procedure followed to attribute the blueshift of the band gap to quantum confinement of the valence band is appropriate, we compare, in Fig. 3, the band structure calculated for 2 and 4 DL with that obtained by performing a calculation of a supercell containing both 2 and $4 \mathrm{DL}$ of $\mathrm{Si}$ separated by $\mathrm{CaF}_{2}$ [24]. In order to help identify the levels we have also shown the amount of $s$ and $p$ wave function located on the inner Si plane for the 2 DL [Fig. 3(a)] and for the 4 DL [Fig. 3(b)]. The calculation for the composite sample containing both 2 and $4 \mathrm{DL}$ is shown twice, reporting the amount of wave function located on the inner Si plane of the 2 and 4 DL [Figs. 3(c) and 3(d), respectively]. It can be seen, by comparing the latter two figures, that the topmost double degenerate valence state of the composite sample is localized in the $4 \mathrm{DL}$ [note also the resemblance of the composition and dispersion of this state with the related one in Fig. 3(b)] while the successive one is localized in the 2 DL [see also Fig. 3(a)]. The energy separation between these two states is exactly the same as that obtained (and shown in Fig. 2) by aligning the fluorine $2 s$ core level.

The position of the interface states in the composite sample, conversely, is not simply the superposition of those of the $2 \mathrm{DL}$ and $4 \mathrm{DL}$; as a consequence, for the composite sample, the minimum direct gap becomes only $0.23 \mathrm{eV}$ higher than the indirect one, and occurs at the $\Gamma$ point.

In order to understand why, in Fig. 3 we have also plotted the dispersion along the (111) direction $Z$ orthogonal to the interfaces. The Ca-Si bonding-antibonding interface states have a finite dispersion along this direction, contrary to those related to the $\mathrm{Si}$ valence band which are completely flat due to their confined character. Further evidence of the localization around the interface of these 

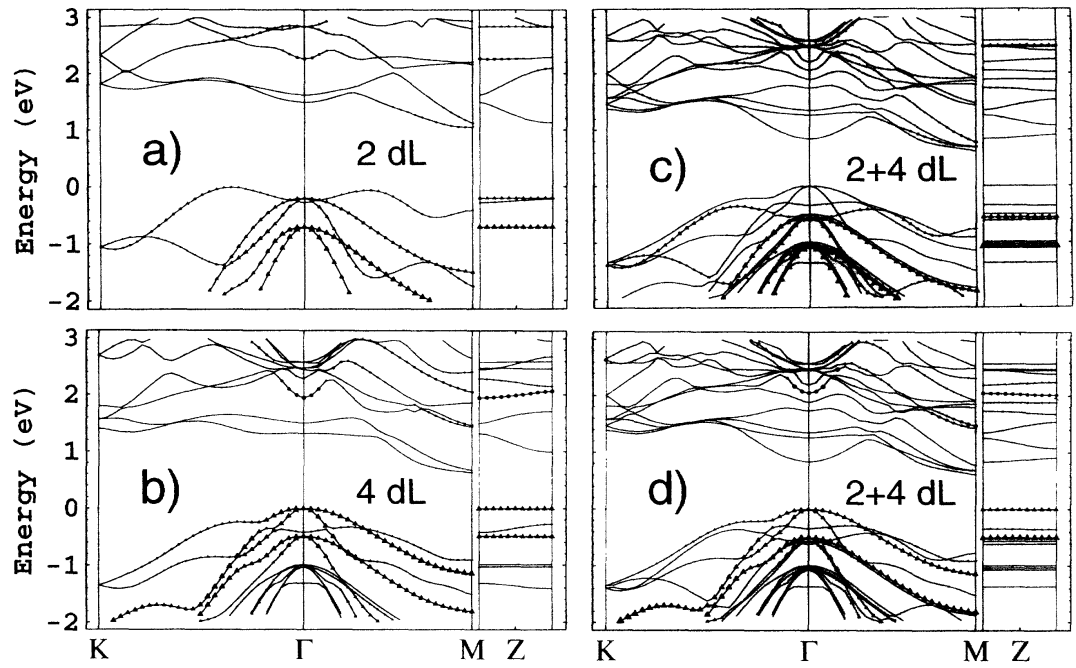

FIG. 3. Band structure projected along two symmetry directions of the hexagonal two-dimensional BZ of the (111) surface ( $\Gamma-M$ and $\Gamma-K$ reaching out one corner and the middle of one side of the hexagon, respectively) and along the (111) direction perpendicular to the surface $\mathrm{BZ}$, indicated as $Z$. (a) For $2 \mathrm{DL} \mathrm{Si}$ in $\mathrm{CaF}_{2}$; the dimension of dots and triangles show, respectively, the amount of the $s$ and $p$ components of the wave function located on the inner Si plane; (b) as in panel (a) for $4 \mathrm{DL} \mathrm{Si}$ in $\mathrm{CaF}_{2}$; (c) for the $2+4 \mathrm{DL}$ composite sample showing the amount of wave function located on the inner Si plane of the $2 \mathrm{DL}$; (d) as in panel (c) for the inner Si plane of the $4 \mathrm{DL}$. Energies (in eV) are referred to the valence band maximum.

states, and of the confined nature of the state related to the bulk valence band, comes from the calculated wave function at the $\Gamma$ point plotted along the growth direction $Z$ shown in Fig. 4. This figure shows, furthermore, that away from the extremal points, the interface states are, strictly speaking, resonant states whence their large spread away from the interface. This makes that only for very thick $\mathrm{CaF}_{2}$ layers, not accessible by our calcu-

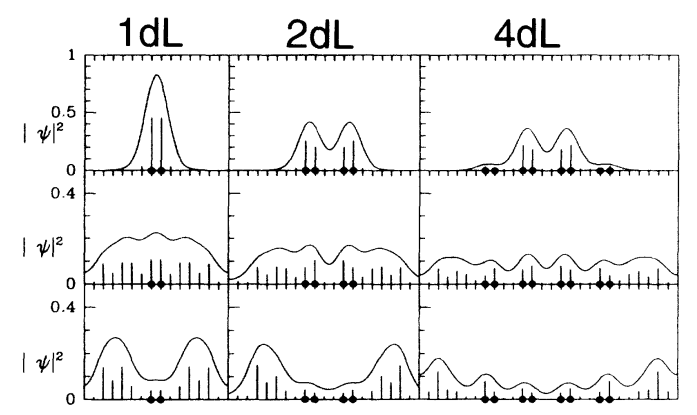

FIG. 4. Squared amplitude of the wave function along the (111) growth direction. The dots indicate the position of the Si planes. The envelope is obtained by dressing the squared amplitude of the wave function on each plane (indicated by the vertical bars) with a Gaussian, two interplane spacing wide. Top panels: double degenerate top valence state at $\Gamma$ for 1,2 , and $4 \mathrm{DL} \mathrm{Si}$ in $\mathrm{CaF}_{2}$; note the confined character. Middle panels: same for the upper valence interface state at $\Gamma$; this state is spread over both the Si layer and the interface. Lower panels: same for the lowest conduction state at $\Gamma$; note the pronounced interface character. lation, the interface states of the 2 and $4 \mathrm{DL}$ can be decoupled. The coupling due to the finite $\mathrm{CaF}_{2}$ thickness leads, as anticipated, to a conduction state at $\Gamma$ only 0.23 $\mathrm{eV}$ higher than the minimum occurring at the $M$ point, i.e., leads to a quasidirect band structure. The position of this interface state can be further lowered by choosing thinner $\mathrm{CaF}_{2}$ layers. We do not pursue this line at this stage but we suggest that by carefully choosing the thickness and the stacking of $\mathrm{Si}$ and $\mathrm{CaF}_{2}$ layers a new, strong contribution to radiative recombination comes into play. In fact, the transition at $\Gamma$ from the lowest interface state to the uppermost valence band is dipole allowed, due to the $s$ character of the Ca-derived interface state and $p$ character of the Si-derived state (see also Figs. 3 and 5), and has a finite spatial overlap due to the penetration of the resonant interface state into the $\mathrm{Si}$ layer.

For completeness we show in Fig. 5 the localization of the energy level for the $4 \mathrm{DL}$ on the interfacial $\mathrm{Si}$ and Ca planes. It can be seen that, while around $\Gamma$ the interface states are mostly localized on the $\mathrm{Ca}$ atoms, at finite wave vectors the weight is almost completely on the interfacial Si planes. The empty and filled interface states run parallel to each other resulting in a high joint density of states. By looking at the $s$ and $p$ composition of the states around the gap, shown in Figs. 5 and 3, one can see that there are a wealth of dipole allowed transitions both at $\Gamma$ and at finite wave vectors.

In conclusion, we have calculated the electronic properties of thin $\mathrm{Si}(111)$ slabs in $\mathrm{CaF}_{2}$, a system which, due to its well characterized structure, is an ideal testing ground for experimental and theoretical comparisons. We have 


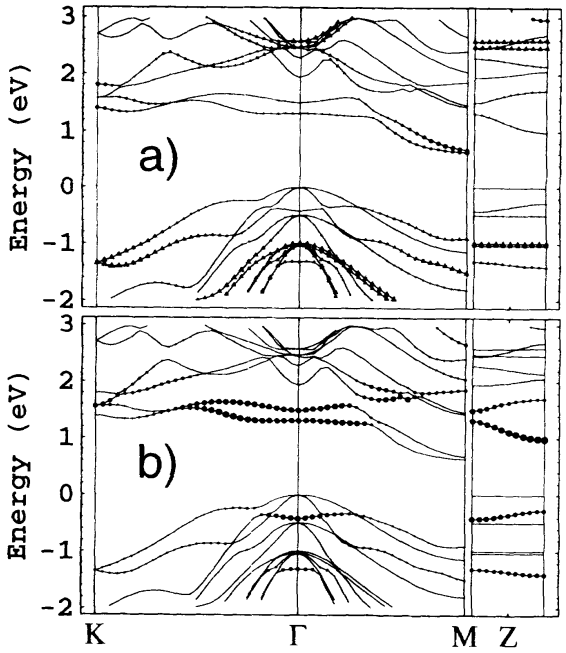

FIG. 5. Band structure as in Fig. 3 for $4 \mathrm{DL} \mathrm{Si}$ in $\mathrm{CaF}_{2}$. (a) The dimension of dots and triangles show, respectively, the amount of the $s$ and $p$ components of the wave function located on the interface Si plane; (b) as in panel (a) for the interface Ca plane.

shown that confinement of the valence band dominates the Si band gap opening, while Si-Ca hybridization effects lead to dipole allowed optical transition all over the BZ. In analogy to $p$-Si $[7,10,12,13]$ the variety of possible low energy interband transitions might lead to visible radiative recombination. Furthermore, we have shown that a quasidirect band gap may be achieved for structures composed of $\mathrm{Si}$ layers of different thicknesses. A calculation of dipole matrix elements for optical transitions is in progress. We hope that our results will stimulate further experimental work on this system.

This work has been carried out within the European projects ESPRIT III No. 7228 EOLIS (S.O. and F.B.) and No. 7260 SOLDES (A.F.). We thank O. Bisi for fruitful discussions.

[1] L. T. Canham, Appl. Phys. Lett. 57, 1046 (1990).

[2] For a recent review see L. T. Canham, in Optical Properties of Low Dimensional Silicon Structures, edited by D. Bensahel, L. T. Canham, and S. Ossicini (Kluwer Aca- demic Publishers, Dordrecht, 1993), p. 81.

[3] M. S. Hybertsen, Mater. Res. Soc. Proc. 256, 179 (1992).

[4] J. P. Proot, C. Delerue, and G. Allan, Appl. Phys. Lett. 61, 1948 (1992).

[5] S. Y. Ren and J. D. Dow, Phys. Rev. B 45, 6492 (1992).

[6] G. D. Sanders and Y. C. Chang, Phys. Rev. B 45, 9202 (1992).

[7] F. Koch, V. Petrova-Koch, T. Muschik, A. Kux, F. Müller, V. Gavrilenko, and F. Möller, in The Physics of Semiconductors, edited by Ping Yang and Hou-Zhi Zheng (World Scientific, Singapore, 1992), p. 1483.

[8] A. J. Read, R. J. Needs, K. J. Nash, L. T. Canham, P. D. J. Calcott, and A. Qteish, Phys. Rev. Lett. 69, 1232 (1992).

[9] F. Buda, J. Kohanoff, and M. Parrinello, Phys. Rev. Lett. 69, 1272 (1992).

[10] T. Ohno, K. Shiraishi, and T. Ogawa, Phys. Rev. Lett. 69, 2400 (1992).

[11] C. G. Van de Walle and J. E. Northrup, Phys. Rev. Lett. 70, 1116 (1993).

[12] M. S. Hybertsen and M. Needels, Phys. Rev. B 48, 4608 (1993).

[13] P. D. J. Calcott, K. J. Nash, L. T. Canham, M. J. Kane, and D. Brumhead, J. Phys. Condens. Matter 5, L91 (1993).

[14] J. C. Alvarez, K. Hirano, A. Yu Kazimirov, M. V. Kovalchuk, A. Ya Kreines, N. S. Sokolov, and N. L. Yakoviev, Semicond. Sci. Technol. 7, 1432 (1992).

[15] M. A. Olmstead and R. D. Bringans, J. Electron Spectrosc. Relat. Phenom. 51, 599 (1990).

[16] J. Zegenhagen and J. R. Patel, Phys. Rev. B 41, 5315 (1990).

[17] R. M. Tromp and M. C. Reuter, Phys. Rev. Lett. 61, 1756 (1988).

[18] A. P. Taylor, K. Stokes, P. D. Persons, L. J. Schowalter, and F. K. Legoues, Proceedings of MRS Conference, 1992, Boston (to be published).

[19] S. Ossicini, C. Arcangeli, and O. Bisi, Phys. Rev. B 43, 9823 (1991); Surf. Sci. 269/270, 743 (1992).

[20] H. Fujitani and S. Asano, Surf. Sci. 268, 265 (1992).

[21] F. Arnaud D'Avitaya (private communication).

[22] M. Buongiorno Nardelli, F. Finocchi, M. Palummo, R. De Felice, C. M. Bertoni, F. Bernardini, and S. Ossicini, Surf. Sci. 269/270, 879 (1992).

[23] F. Bernardini, A. Fasolino, and S. Ossicini (to be published).

[24] The $39.4 \AA$ thick supercell of the composite sample is constituted of: $2 \mathrm{Si} \mathrm{DL}-3 \mathrm{CaF}_{2}$ planes - $4 \mathrm{Si} \mathrm{DL}-4$ $\mathrm{CaF}_{2}$ planes. 